\title{
MISTAKES IN CHRONIC CONSTIPATION TREATMENT IN ADULTS
}

\section{Pérez Aisa Á}

Agencia Sanitaria Costa del Sol. Marbella, Málaga.

\section{Resumen}

El objetivo de este tema es resumir, condensar todos los conocimientos en el enfoque terapéutico y poner en evidencia alguna situación más especial que nos podemos encontrar en el contexto del estreñimiento crónico funcional en el adulto. Para ello utilizaremos el planteamiento de escenarios clínicos con casos habituales en la práctica clínica que nos permitirán insistir en el manejo terapéutico de las diferentes situaciones clínicas.

Palabras clave: estreñimiento, tratamiento, errores.

\section{Abstract}

The objective of this theme is to summarize, condense all the knowledge related to treatment and also to highlight some more special situation that we can find in the context of chronic functional constipation in adults. For this, we will use the approach of clinical scenarios with habitual cases in clinical practice that will allow us

\section{CORRESPONDENCIA \\ Ángeles Perez Aisa \\ Agencia Sanitaria Costa del Sol \\ 29602 Marbella (Málaga) \\ drapereza@hotmail.com}

Fecha de envío: 03/11/2019

Fecha de aceptación: 14/12/2020 to insist on the therapeutic management of the different clinical situations.

Keywords: constipation, treatment, mistakes.

\section{Escenario clínico I}

Presentamos el caso de una mujer de 52 años que refiere como antecedentes familiares a su madre con una enfermedad de Alzheimer y como antecedentes personales presenta hipertensión arterial que controla con atenolol 50 e hidroclorotiazida, hipercolesterolemia para lo que toma simvastatina. También refiere un síndrome ansioso-depresivo por lo que toma lorazepam por las noches. Se trata de una paciente con una obesidad tipo 2. Acude a la consulta por presentar síntomas de estreñimiento de más de diez años evolución. Refiere heces de pequeño tamaño, frecuentemente duras y un gran esfuerzo defecatorio de tal forma que tienen que acudir en varias ocasiones al baño aunque la mayoría de veces no consigue defecar o expulsa sólo una pequeña parte. Necesita ayudarse con los dedos y siempre se queda insatisfecha. Nos relata que en alguna ocasión y coincidiendo con esfuerzo defecatorio expulsión de sangre roja de escasa cuantía. En el interrogatorio dirigido nos relata un dolor pélvico que define como sensación de peso que le dura todo el día y que empeora después del esfuerzo defecatorio.

En la exploración física observamos hemorroides externas, en el canal anal se aprecia buena presión basal y durante la 
contracción voluntaria. En el tacto rectal la ampolla rectal está vacía, sin masas, no se palpa rectocele pero es doloroso, especialmente a la palpación del músculo puborrectal que reproduce el dolor postdefecatorio que la paciente refiere tener habitualmente. Le invitamos a realizar un test expulsivo simulado y este resulta compatible con una disinergia anorrectal. En este caso no nos planteamos ninguna prueba complementaria inicial adicional y nos decidimos por una estrategia positiva puesto que tenemos una paciente con síntomas de larga data sin datos de alarma y $\sin$ factores de riesgo especiales ${ }^{1}$. claves $^{2}$ :

Iniciamos nuestra estrategia terapéutica y en ella son

\section{- Definir objetivos claros}

\section{- Pautar fármacos cuya posología o forma galénica sea simple.}

- Facilitar al paciente la información y recordatorios escritos, simples y de fácil comprensión.

- Elaborar "calendarios" de cumplimiento mediante los cuales monitorizar la toma de medicación o la realización de actividades pautadas.

- Incluir en todas estas estrategias a los familiares y cuidadores sobre todo en el caso de pacientes ancianos o dependientes

\section{- Establecer la regularidad en el tratamiento indicado para el estreñimiento.}

En esta tarea el personal de enfermería puede desempeñar un papel muy eficaz en la educación sanitaria y en el seguimiento de estos pacientes.

El tratamiento va a pivotar en tres elementos claves como son una serie de consejo sobre hábitos alimentarios, la utilización de laxantes que hayan demostrado eficacia con un adecuada posología y unas recomendaciones en relación a la corrección postural y esfuerzo defecatorio. En este último aspecto en el escenario clínico que nos ocupa recomendar a nuestra paciente adoptar una posición en cuclillas o con un escabel a la hora de la defecación podría modificar el ángulo que genera el músculo puborrectal relajando este y facilitando la evacuación.

Las medidas higiénico dietéticas han sido revisadas de forma completa en el apartado relativo al primer escalón terapéutico del estreñimiento funcional ${ }^{3}$. Sólo insistiremos en algunos aspectos como son en garantizar la ingesta hídrica diaria de 1,5-2 litros de líquidos diarios pero no en mayor cuantía. Recomendaremos el aumento de la fibra diaria a 25-30 g/día pero no en mayor cuantía puesto que la fermentación podría aumentar si existe un tránsito colónico lento y también recordar e insistir en que sea un aporte gradual con incrementos de pequeña cantidad diaria para garantizar tolerabilidad y adaptación del paciente a esta estrategia.

Una de las medidas más eficaces y que deberíamos de implementar de forma activa desde el inicio del manejo de esta condición es la realización de ejercicio aeróbico diario. Existe evidencia de que se mejora el tiempo de tránsito colónico total y a nivel de recto-sigma a la vez que mejora la distensión abdominal y la retención de gas ${ }^{4}$. Por último, en relación con la prescripción de laxantes en el primer escalón de recomendación deberían estar los laxantes osmóticos. Dentro de este grupo incluimos polietilenglicol, sales de magnesio y lactulosa. La evidencia ${ }^{5}$ señala a polietilenglicol como el de elección por su eficacia, buena tolerabilidad y su perfil de seguridad puesto que puede ser usado sin inconvenientes en ancianos, embarazo, insuficiencia hepática y en insuficiencia renal.

En nuestro escenario clínico implementamos todas las medidas referidas con un adecuado cumplimiento por parte de nuestra paciente pero nos encontramos con la realidad, por otro lado muy habitual, de que no se produce la mejoría deseada. En este momento nos plantearemos el algoritmo diagnóstico planteado por Mearin et al. y recogida en la (Figura 1).

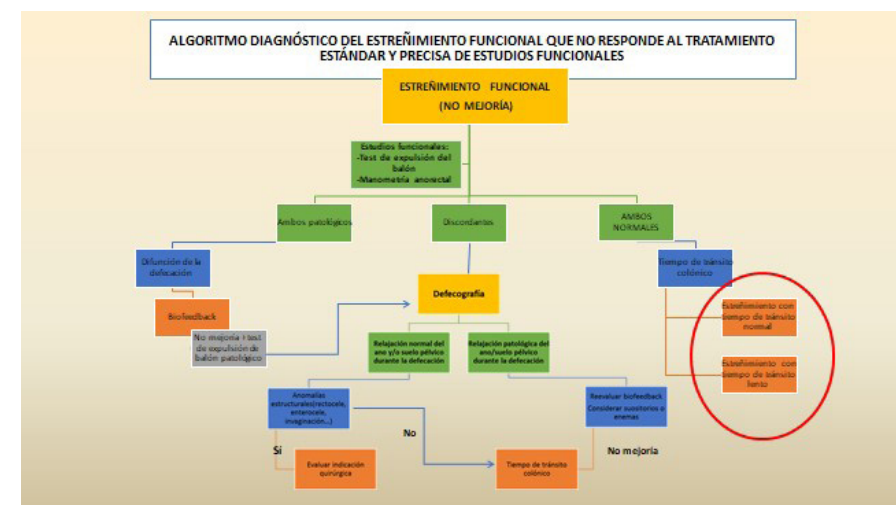

Figura 1

Algoritmo diagnóstico del estreñimiento funcional no respondedor a primer escalón terapéutico.

Se plantea la realización de pruebas complementarias adicionales para explorar la motilidad anorrectal como son la manometría anorrectal y el test de expulsión que se describen de forma amplia en el tema correspondiente ${ }^{6}$. En nuestro caso ambas pruebas presentan resultados patológicos poniendo de manifiesto una maniobra defecatoria paradójica con un esfuerzo insuficiente y un test expulsivo que no se supera a los 60 segundos con un volumen de $60 \mathrm{ml}$. La defecación disinérgica es un trastorno funcional anorrectal que afecta al $40 \%$ de los pacientes con estreñimiento crónico funcional y que para ser corregido precisa un abordaje diferente. Nuestro enfoque terapéutico en estos momentos aunque se deben mantener las medidas descritas previamente, debe de incorporar la rehabilitación o biofeedback anorrectal. Esta estrategia también ha sido ampliamente comentada en un tema explícito pero vamos a hacer hincapié en algunos factores claves. El objetivo del biofeedback en los pacientes con disinergia defecatoria es restablecer un patrón defecatorio normal, consiguiendo una contracción abdominal adecuada junto con la relajación de los esfínteres. Las anomalías a corregir son la contracción anal paradójica, la relajación anal incompleta, el esfuerzo insuficiente y la hiposensibilidad rectal ${ }^{7}$. Las indicaciones para iniciar este tratamiento rehabilitador son que se cumplan criterios diagnósticos y que exista una falta de respuesta al tratamiento convencional del estreñimiento crónico funcional. Los objetivos terapéuticos que nos plantearemos serán en caso de hipertonía basal, indicar ejercicios de relajación anal y control diafragmático de la respiración y del esfuerzo defecatorio, conseguir 
una maniobra defecatoria coordinada, contracción abdominal adecuada, relajación anal y mejorar la percepción rectal ${ }^{8,9}$. Existen estudios comparativos en los que la rehabilitación es superior a placebo, dieta, otras técnicas de biofeedback simulado, diazepam y laxantes consiguiendo unos resultados favorables en el $50-86 \%$ pacientes según los estudios y con una media $69 \%$. Existen unos factores de buen pronóstico como son la presencia de heces duras, que el paciente adopte una favorable disposición a participar, la existencia de presión de reposo elevada o bien una prueba expulsiva balón rectal prolongada (sensibilidad 0,79 , especificidad 0,81 ). Sin embargo, el biofeedback no está indicado en pacientes con estreñimiento crónico por tiempo de tránsito colónico prolongado consiguiendo peores resultados en aquellos pacientes con un tratamiento previo prolongado con laxantes. El biofeedback ha demostrado en varios estudios que mejora calidad de vida y de los síntomas abdominales asociados ${ }^{10,11}$.

Como mensajes claros que podemos aprender de este escenario clínico es que debemos implementar todas las estrategias para establecer un adecuado diagnóstico. Como base del tratamiento debemos insistir en las medidas de primer escalón que realmente han demostrado eficacia (ejercicio, polietilenglicol, etc.) y deberemos plantear tratamiento específico con biofeedback de defecación en los pacientes con disinergia defecatoria sin obstinarnos en laxantes sin corregir la defecación.

\section{Escenario clínico 2}

Se presenta el caso de una mujer de 29 años embarazada que presenta dificultad para la defecación. En la anamnesis nos refiere episodios previos de estreñimiento más o menos severos a lo largo de su vida que han empeorado al quedarse embarazada y reconoce que para corregirlo utilizaba hierbas que compraba en herbolario pero embarazada no se ha atrevido a tomarlas. Sus deposiciones corresponden a un tipo 2 de la escala de Bristol y nos refiere que precisa de mucho tiempo en el baño para conseguir defecar al igual que la necesidad de digitalizarse conseguiendo defecación que en raras ocasiones es satisfactoria.

Nos encontramos en un escenario clínico con algunas peculiaridades puesto que durante el embarazo se producen una serie de factores predisponentes ${ }^{12}$ :

- Existe una disminución de la motilidad intestinal bajo la influencia de la progesterona y disminución de la motilina.

- El tránsito colónico se encuentra enlentecido.

- Por los cambios propios de la pelvis existen unos cambios en dinámica anorectal.

- Empeora con la administración de suplementos de hierro y calcio que son fármacos que en muchas ocasiones se utilizan durante el embarazo.

- Muchas mujeres viven una falta de movilidad por patologías asociadas como son las lumbalgias.
- Se produce una disminución de la ingesta hídrica y una redistribución de esta.

Como consecuencias del estreñimiento no corregido debemos destacar que se puede producir una lesión perineal durante el embarazo con el consiguiente sufrimiento de suelo pélvico, hasta un $45 \%$ de las mujeres pueden desarrollar hemorroides y debemos de tener en cuenta que todas estas circunstancias se pueden poner de manifiesto de una forma más relevante durante el tercer trimestre ${ }^{12}$.

Por otro lado debemos valorar que el estreñimiento funcional durante el embarazo tiene unas connotaciones especiales puesto que por muchas mujeres y facultativos el estreñimiento se considera algo normal e inherente al embarazo. No podemos considerar que sea una enfermedad sino un síntoma o una consecuencia de los cambios fisiológicos que provoca el embarazo, y muchas veces también de unos malos hábitos higiénico-dietéticos. Se añade que no hay un criterio diagnóstico claro dejándose a la capacidad de la mujer para aguantar o considerarlo incómodo como el momento de consulta por esta situación y por último tanto embarazadas como profesionales son reticentes a los fármacos en el embarazo.

En el abordaje diagnóstico el enfoque no varía de otras situaciones por lo que debemos de plantearnos las preguntas claves para el correcto diagnóstico, deberíamos evidenciar complicaciones como son hemorroides, prolapso o fisura anal y si es posible reducir consumo de fármacos favorecedores de estreñimiento.

Los criterios derivación a Atención Especializada:

- Sospecha de estreñimiento secundario a enfermedad orgánica: sangre mezclada heces, pérdida peso o ausencia de ganancia, dolor abdominal y/o tenesmo.

- Riesgo de lesión perineal asociada a estreñimiento.

- Antecedentes de incontinencia anal postparto.

- En el algoritmo terapéutico (Figura 2) debemos destacar las consideraciones de retirada de fármacos y la valoración de disinergia defecatoria rehabilitable puesto que durante los primeros trimestres no hay contraindicación para plantear biofeedback de defecación. Si no estamos en esa situación el tratamiento inicial se basa en medidas generales como ejercicio físico adecuado y aumento de la ingesta hídrica. El polietilenglicol (PEG) es el laxante osmótico con mejor perfil de seguridad y de primera elección en este contexto. En caso de no existir respuesta podemos utilizar laxantes estimulantes o bien enemas de limpieza para corregir los casos más reticentes.

Como mensajes claves que podemos recoger de este escenario clínico debemos destacar la importancia de manejar bien este síntoma durante el embarazo por consecuencias posteriores a nivel suelo pélvico. Tener presente que disponemos de exploraciones diagnósticas seguras en embarazo para disinergia y tratamiento rehabilitador, fármacos seguros y un algoritmo lógico de manejo. 


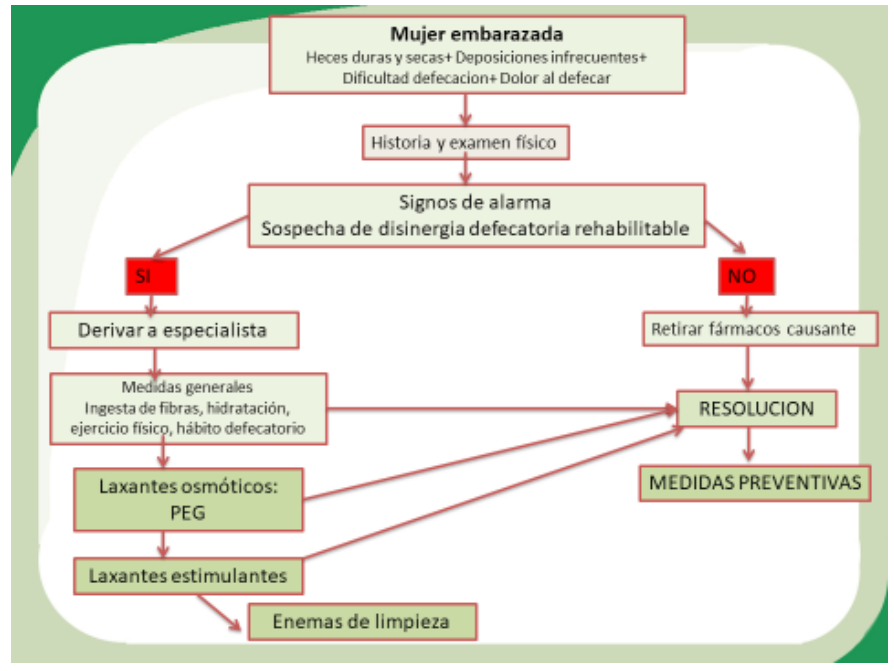

Figura 2

Algoritmo diagnóstico de estreñimiento en embarazo.

\section{Escenario clínico 3}

Se trata de un varón de 83 años que presenta como antecedentes patológicos: HTA, DM desde hace doce años, insuficiencia renal en estadio $3 \mathrm{~B} 1$, con filtrado glomerular moderadamente disminuido $\left(30-59 \mathrm{~mL} / \mathrm{min} / 1,73 \mathrm{~m}^{2}\right)$ e hipertrofia prostática. Su tratamiento habitual consiste en: AAS-diltiazemsulfato ferroso-glibenclamida. Su movilidad es limitada y se encuentra institucionalizado en una residencia de la tercera edad. Es remitido a Urgencias por presentar diarrea con manchado de pañales y distensión abdominal desde hace 48 horas. Su hábito intestinal previo consiste en una deposición cada 4-5 días Bristol 1-2 con necesidad de laxantes (Plantago ovata). En la exploración física destaca un regular estado general con distensión abdominal y dolor a la palpación difusa. Se le realizan las medidas más básicas y en el tacto rectal destaca la presencia de colgajos cutáneos y restos fecales perianales y a punta de dedo se palpa un fecaloma. Se decide la realización de radiografía de abdomen que evidencia la presencia de fecaloma con asas discretamente dilatadas.

La impactación fecal ${ }^{13,14}$ se define como la acumulación de heces en el colon que el paciente es incapaz de evacuar por sí mismo, permaneciendo un mayor tiempo en el colon y recto siendo sometidas a la absorción de agua de forma mantenida. Se asocia a una disminución de la sensación rectal y puede acompañarse de incontinencia fecal con pseudodiarrea mediada por una mayor producción de moco a nivel rectal como respuesta a la existencia de una masa fecal indurada. Su prevalencia no es bien conocida en población general pero algunos datos estiman que en el medio residencial más de un $>50 \%$ de pacientes con antecedentes de estreñimiento crónico la pueden desarrollar.

Existen una serie de condiciones de riesgo inherentes al paciente como son la alta comorbilidad, el consumo de fármacos, inamovilidad establecida en los ancianos, el deterioro cognitivo/ demencia y procesos neurológicos (ictus, Parkinson, disautonomía) que empeoran la situación previa. Pero también existen unos factores inherentes al manejo del estreñimiento crónico en este tipo de pacientes como son el empleo de laxantes formadores de bolo en pacientes inmovilizados, el insuficiente seguimiento de la respuesta al tratamiento laxante y la escasa sensibilización hacia el desarrollo de impactación fecal.

La inmovilidad es uno de los factores donde pivotan muchos de los demás ${ }^{13}$. Condiciona una disminución de la ingesta tanto de alimentos como de agua y de fibra lo que conlleva a la conversión de las heces en una consistencia dura, esta consistencia se ve favorecida por la disminución del peristaltismo y un aumento de la absorción de agua intestinal. Es importante resaltar que la inmovilidad establece la necesidad de utilizar absorbentes (pañales) que junto a la falta de privacidad, baños compartidos, falta de tiempo, ambiente desfavorable con poca intimidad, posición horizontal en los pacientes encamados hacen que la defecación no transcurra con las condiciones básicas de confort.

Se pueden producir complicaciones por efecto sobre la pared intestinal como son la colitis estercorácea, la úlcera estercorácea, perforación y/o megacolon. Y también pueden presentarse complicaciones mecánicas como son la pseudodiarrea, la suboclusión intestinal y la obstrucción intestinal. No hay que desdeñar otras complicaciones sobre estructuras próximas como son compresión de la vejiga urinaria, uropatía obstructiva y/o infecciones urinaria.

El algoritmo terapéutico se resume en la figura 3.

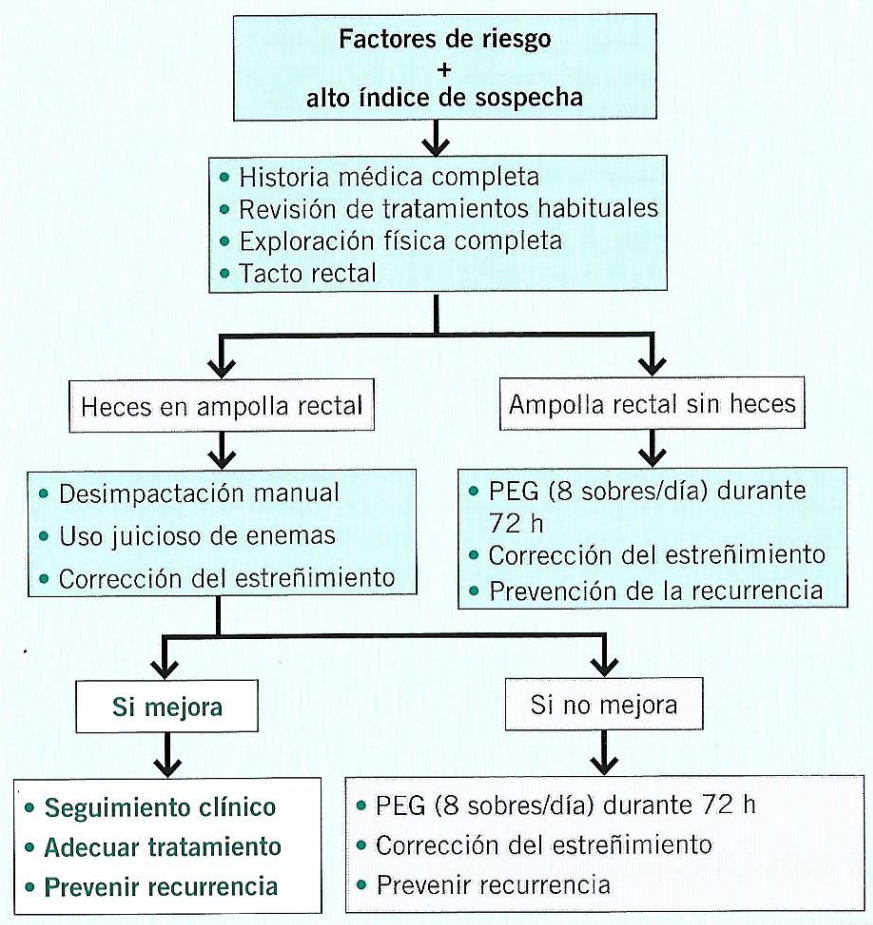

Figura 3

Algoritmo terapéutico en impactación fecal en el anciano.

Como mensajes de utilidad en este escenario clínico debemos considerar que la impactación fecal afecta a pacientes muy frágiles con grandes factores de riesgo, que debemos implementar una adecuada exploración física y alta sospecha. Se debe plantear un enfoque terapéutico adecuado para evitar consecuencias graves y el polietilenglicol (PEG) es el fármaco de elección en el tratamiento ${ }^{15}$. 


\section{Bibliografía}

1. Lacy BE, Mearin F, Chang L, et al. Bowel disorders. Gastroenterology. 2016;150(6):1393-1407e5. doi:10.1053/j.gastro.2016.02.031

2. Serra J, Mascort-Roca J, Marzo-Castillejo M, et al. Guía de práctica clínica sobre el manejo del estreñimiento crónico en el paciente adulto. Parte 1: Definición, etiología y manifestaciones clínicas. Gastroenterol Hepatol. 2017;40(3):132-141. doi:10.1016/j.gastrohep.2016.02.006

3. Mearin F, Ciriza C, Rey E, Mascort JJ. Guía de práctica clínica del síndrome del intestino irritable con estreñimiento y estreñimiento funcional en adultos: tratamiento. (Parte 2 de 2). Atención Primaria. 2017;49(3):177-194.

4. Singh P, Staller K, Barshop K, Dai E, Newman J, Yoon S, Castel S, Kuo B. Patients with irritable bowel syndrome-diarrhea have lower disease-specific quality of life than irritable bowel syndrome-constipation. World J Gastroenterol. 2015Jul 14;21 (26):8103-9. doi: 10.3748/wjg.v21.i26.8103.

5. Chapman RW, Stanghellini V, Geraint M, Halphen M. Randomized clinical trial: macrogol/PEG3350 plus electrolytes for treatment of patients with constipation associated with irritable bowel syndrome. Am J Gastroenterol. 2013 Sep;108(9):1508-15. doi: 10.1038/ajg.2013.197. Epub 2013 Jul 9.

6. Mínguez Pérez M, Más Mercader P. Estreñimiento funcional: diagnóstico. In: Lacima Vidal G, Serra Pueyo J, Mínguez Pérez M, Accarino Garaveta A, eds. Tratado de Neurogastroenterología Y Motilidad Digestiva. Madrid: Editorial Médica Panamericana; 2014:87-93

7. Lacima G, Mínguez M. Biofeedback en "Tratado de Neurogastroenterología y Motilidad Digestiva. 2014, Editorial Panamericana. Pp227-246.
8. Baker J, Eswaran S, Saad R, Menees $\mathrm{S}$ et al. Abdominal symptoms are common and benefit from Biofeedback therapy in patients with dysynergic defecation. Clinical Traslational Gastroenterol 2015; 6: e105.

9. Wald A. Constipation. Advances in Diagnosis and Treatment. JAMA. 2016;315(2):185-191

10. Rao SS, Benninga MA, Bharucha AE, Chiarioni G, Di Lorenzo C, Whitehead WE. ANMS-ESNM position paper and consensus guidelines on biofeedback therapy for anorectal disorders. Neurogastroenterol Motil 2015; 27: 594

11. Baker J, Eswaran S, Saad R, Menees S, Shifferd J, Erickson K, Barthelemy A, Chey WD. Abdominal Symptoms Are Common and Benefit from Biofeedback Therapy in Patients with Dyssynergic Defecation. Clin Traslational Gastroenterology 2015; 6: e105

12. Sociedad Española de Ginecología y Obstetricia. "Protocolo de Dagnóstico y tratamiento del estreñimiento durante el emabrazo".

13. Talley NJ, Fleming KC, Evans JM, et al. Constipation in an elderly community: a study of prevalence and potential risk factors. Am J Gastroenterol. 1996;91(1):19-25. http://www.ncbi.nlm.nih.gov/pubmed/8561137. Accessed October 2, 2017

14. Bouras E, Vazquez-Roque M. Epidemiology and management of chronic constipation in elderly patients. Clin Interv Aging. 2015;10:919. doi:10.2147/ CIA.S54304

15. Minguez M, López-Higueras A, Júdez J. Use of polyethylene glycol in functional constipation and fecal impaction. Rev Esp Enferm Dig 2016, 108: 790-806. 